increase in HOMA-IR and a 10\% increase in TSH level was associated with one fold increase in geometric mean of insulin level $(\mathrm{P}=0.003,0.002$, respectively), but the relationship between TSH and triglyceride levels disappeared.

Conclusion TSH level was found related to the glucose metabolism in overweight and obese adolescents. Further prospective studies are needed to clarify the mechanism of this relationship.

\section{P524 FUNCTIONAL DISORDERS OF THE COLON IN ADOLESCENTS WITH OBESITY: ASSESSMENT OF QUALITY OF LIFE AND PHYSICAL ACTIVITY}

Anastasiya Romanitsa, Lyubov Rychkova*. Scientific Centre for Family Health and Human Reproduction Problems, Irkutsk, Russian Federation

\subsection{6/archdischild-2019-epa.859}

Introduction A child's quality of life (QL) is an integral characteristic of physical, psychological and social functioning of a child, based on self-perception and/or other peoples' perception. Obesity affects the QL and often leads to development of comorbid diseases, disabilities and invalidity. The majority of people with obesity face difficulties integrating in the society due to poorer health, physical limitations or psychological issues.

The aim To characterize the change in QL and physical activity (PA) in adolescents with obesity and functional disorders of the colon (FDC).

Materials and methods We examined 111 adolescents with obesity: 64 girls and 47 boys (mean age 14.5 \pm 2.0 years), from 2016 to 2018. The main group included 73 adolescents with obesity, who suffered from irritable bowel syndrome (IBS). The second group included adolescents with obesity and other FDC, the third group comprised adolescents with obesity without FDC. All children underwent the questionnaire survey to establish their QL and PA (PAQ-C and PAQ-A 1017; PedsQL-4.0). Statistical processing of results was conducted with Statistica 10.0, Windows. Difference was significant at $\mathrm{P}<0.05$.

Results The majority of adolescents with obesity are children with FDC (86.5\%): functional diarrhea $15.6 \%$ and constipation $5.2 \%$, nonspecific bowel disorders $3.1 \%$, and IBS $76.1 \%$, (among them: IBS with constipation - 65.8\%, with diarrhea $13.7 \%$. mixed type $-20.5 \%)$. FA of children 1 and 2 groups $(1.99 \pm 0.56)$, in the 3 rd group $(2.46 \pm 0.82)$. According to the PedsQ questionnaire for adolescents, group 1 had significantly lower total scores of $\mathrm{QL}$ compared to the group 2 (P $=0.03)$, and group $3(\mathrm{P}<0.00)$, statistically significant differences were achieved mainly due to a decrease in emotional functioning $(P=0.0003)$ and social functioning $(P=0.002)$. The percentage of QL in children with other FDC (group 2) is lower compared to group $3(\mathrm{P}<0.01)$, but higher than in the group of adolescents with IBS $(\mathrm{P}=0.002)$.

Conclusions Every 6th obese adolescent has FDCs (86.5\%), among which IBS is most common - 76.1\%. Definitely, QL of adolescents with obesity without FDC is higher than among adolescents with FDC (QL decrease is associated with IBS), mainly due to a decrease in emotional and social functioning. When assessing the relative frequency of changes in QL in children with FDC, significant towards a decrease in school functioning. Obese adolescents with FDC have a 1.2 times lower PA than obese adolescents without FDC.

\section{P525 HOW HIGH IS THE BMI? OVERCOMING THE BARRIERS TO ADDRESSING OBESITY IN THE PAEDIATRIC OUTPATIENT POPULATION}

Rhiannon McBay-Doherty*, Eamonn Sweeney, Karen Orr, Bernadette O'Connor, Mugilan Anandarajan, Anne-Marie McClean. Ulster Hospital Dundonald, Belfast, UK

\subsection{6/archdischild-2019-epa.860}

Background Childhood obesity negatively impacts physical health, mental wellbeing and shortens life expectancy. A 2017 audit of 87 children attending our district hospital's outpatient clinics (OPC) and rapid response unit (RRU) revealed: $15 \%$ of children were obese and $13 \%$ were overweight. $86 \%$ of overweight/obese children did not have their unhealthy weight addressed by a healthcare professional. Focus groups and questionnaires indicated staff often felt underprepared and apprehensive about addressing childhood obesity. Staff sometimes found it difficult to recognise if a child was overweight from raw height/weight data.

Aims To increase childhood obesity recognition by ensuring that $50 \%$ of children will have Body Mass Index (BMI) calculated and plotted and to maintain this practice.

To increase staff knowledge, willingness and confidence to address childhood obesity with families.

Methods IHI QI methodology was used to introduce BMI plotting to RRU \& OPC; beginning in October 2017. A multidisciplinary team including dieticians, physiotherapists, psychologists, nurses and doctors was established. Plan-Do-StudyAct cycles introduced practical changes including I-pads with an app for calculating BMI centiles. Staff received motivational interviewing training to have these crucial conversations with families. Staff and parent feedback were collected through questionnaires.

Results BMI plotting was successfully introduced; going from a median of $0 \%-100 \%$ for both OPC and RRU and increasing the recognition of overweight/obese children to $100 \%$ by 3 months. At 12 months; BMI plotting in OPC was 60\%; higher than our initial aim but showing room for improvement. $100 \%$ of staff who attended multidisciplinary training 'agreed'/'strongly agreed' that it improved their willingness, confidence and preparedness to address obesity with families. At 12 months after intervention, 67\% of overweight and obese children who had their BMI plotted had their weight concerns addressed during their outpatient appointment. 100\% of 27 parents who provided feedback were supportive of the project and $100 \%$ stated that doctors have a duty to raise obesity with families.

Conclusions This QI project has shown that introducing routine BMI plotting and providing staff with training to address obesity with families improves the recognition and response to children who are overweight/obese. We shared our innovative practice by hosting a regional paediatric obesity awareness day for all Northern Irish healthcare professionals; this included a motivational interviewing training session for all staff. Parents share our vision that all overweight/obese children are recognised and supported by healthcare professionals to achieve a healthier weight. 